\title{
SOME MEAN SQUARES OF ENTIRE FUNCTIONS
}

\section{LOUIS DE BRANGES}

Let $E(z)$ be an entire function of exponential type without zeros in the closed upper half plane, $y \geqq 0$, such that

$$
\int \frac{\log ^{+}|E(t)| d t}{1+t^{2}}<\infty
$$

and

$$
\limsup _{y \rightarrow-\infty}|y|^{-1} \log |E(i y)| \leqq \limsup _{y \rightarrow+\infty} y^{-1} \log |E(i y)| .
$$

Such functions sometimes appear in applications of analytic function theory to Hilbert space-for example, $E(z)=\exp (-i z)$.

THEOREM. There is at most one real number $\alpha$ modulo $\pi$ such that the following statement is not true. If $F(z)$ is an entire function of exponential type such that

$$
\int \frac{\log ^{+}|F(t)| d t}{1+t^{2}}<\infty
$$

and

$$
F(i y)=o(E(i|y|))
$$$$
(|y| \rightarrow \infty)
$$

then

$$
\int\left|\frac{F(t)}{E(t)}\right|^{2} d t=2 \pi i \sum \frac{|F(t)|^{2}}{E(t) \bar{E}^{\prime}(t)-\bar{E}(t) E^{\prime}(t)}
$$

where in the sum on the right $t$ ranges in the real numbers such that $e^{i \alpha} E(t)$ is real.

It follows by Boas [1, pp. 83 and 97] that the theorem is vacuous if $E(z)$ is a constant. In what follows we suppose that $E(z)$ is not a constant. We will see in Lemma 2 below that for real $t$,

$$
i\left(\bar{E}(t) E^{\prime}(t)-E(t) \bar{E}^{\prime}(t)\right)>0 .
$$

The interpretation of the theorem is that the sum converges if and only if the integral converges and then (5) holds.

For each real $\alpha$, let

Received by the editors October 4, 1958. 


$$
\begin{aligned}
& S(\alpha, z)=\frac{1}{2} i e^{i \alpha} E(z)-\frac{1}{2} i e^{-i \alpha} \bar{E}(\bar{z}), \\
& C(\alpha, z)=\frac{1}{2} e^{i \alpha} E(z)+\frac{1}{2} e^{-i \alpha} \bar{E}(\bar{z}) .
\end{aligned}
$$

Here $\alpha$ is to be thought of as an index on the entire function of $z$. Note that

$$
\begin{aligned}
& e^{i \alpha} E(z)=C(\alpha, z)-i S(\alpha, z), \\
& \bar{C}(\alpha, \bar{z})=\bar{C}(\alpha, z), \\
& S(\alpha, \bar{z})=\bar{S}(\alpha, z) .
\end{aligned}
$$

Lemma 1. For each real $\alpha$, when $y>0, S(\alpha, z) \neq 0$ and

$$
\operatorname{Im} C(\alpha, z) / S(\alpha, z)<0 .
$$

Lemma 2. For each real $\alpha$, the zeros of $S(\alpha, z)$ are real,

$$
C(\alpha, z) S^{\prime}(\alpha, z)>0 \text { when } S(\alpha, z)=0
$$

and

$$
\sum_{S(\alpha, t)=0} C(\alpha, t)\left[S^{\prime}(\alpha, t)\left(1+t^{2}\right)\right]^{-1}<\infty
$$

For all real $t$,

$$
\begin{aligned}
i\left(\bar{E}(t) E^{\prime}(t)-E(t) \bar{E}^{\prime}(t)\right) & =2 C(\alpha, t) S^{\prime}(\alpha, t)-2 S(\alpha, t) C^{\prime}(\alpha, t) \\
& >0
\end{aligned}
$$

LeMma 3. For every real $\alpha$,

$$
E(i y)=O(y S(\alpha, i y)) \quad(|y| \rightarrow \infty) .
$$

There is at most one real number $\alpha$ modulo $\pi$ such that

$$
S(\alpha, i y)=o(E(i y)) \quad(y \rightarrow+\infty) .
$$

Lemma 4. If $\alpha$ and $\beta$ are real numbers and if $\alpha$ does not satisfy (11), and if $F_{1}(z)$ is an entire function of exponential type satisfying (3) and (4) and such that the right hand side of (5) converges, then

$$
\begin{gathered}
2 i \sum_{S(\alpha, t)=0} \frac{1}{E(t) \bar{E}^{\prime}(t)-\bar{E}(t) E^{\prime}(t)} \frac{F_{1}(t) S(\beta, t)}{t-\lambda} \\
=\frac{2 i F_{1}(\lambda) S^{\prime}(\beta, \lambda)}{E(\lambda) \bar{E}^{\prime}(\lambda)-\bar{E}(\lambda) E^{\prime}(\lambda)}
\end{gathered}
$$

whenever $S(\beta, \lambda)=0$. 
Proof of Lemma 1. By Boas [1, pp. 129-130], when $y \geqq 0,|E(\bar{z})|$ $\leqq|E(z)|$, and since $E(z)$ is not a constant, when $y>0$ the strict inequality holds. Therefore, by (6), when $y>0$,

$$
|C(\alpha, z)+i S(\alpha, z)|<|C(\alpha, z)-i S(\alpha, z)| \text {. }
$$

So when $y>0, S(\alpha, z) \neq 0$ and

$$
|C(\alpha, z) / S(\alpha, z)+i|<|C(\alpha, z) / S(\alpha, z)-i|
$$

and hence

$$
\operatorname{Im} C(\alpha, z) / S(\alpha, z)<0 .
$$

Proof of Lemma 2. By Lemma 1, for each real $\alpha$,

$$
-\operatorname{Im} C(\alpha, z) / S(\alpha, z)
$$

is non-negative and harmonic in the upper half plane $y>0$. By the Poisson representation (Loomis and Widder [4]), there is $A=A(\alpha)$ $\geqq 0$ and a non-negative measure $\mu=\mu_{\alpha}$ on the Borel sets of the real line such that

$$
\int\left(1+t^{2}\right)^{-1} d \mu(t)<\infty
$$

and for $y>0$,

$$
-\operatorname{Im} C(\alpha, z) / S(\alpha, z)=\int \frac{y d \mu(t)}{(t-x)^{2}+y^{2}}+A y .
$$

Differentiating each side with respect to $x$, we get

$$
-\operatorname{Im} \frac{d}{d z} \frac{C(\alpha, z)}{S(\alpha, z)}=\operatorname{Im} \int \frac{d \mu(t)}{(t-z)^{2}} .
$$

Differentiating instead with respect to $y$ and using the CauchyRiemann equations, we get

$$
-\operatorname{Re} \frac{d}{d z} \frac{C(\alpha, z)}{S(\alpha, z)}=\operatorname{Re} \int \frac{d \mu(t)}{(t-z)^{2}}+A .
$$

Therefore,

$$
-\frac{d}{d z} \frac{C(\alpha, z)}{S(\alpha, z)}=\int \frac{d \mu(t)}{(t-z)^{2}}+A .
$$

It follows from this formula that the zeros of $S(\alpha, z)$ are real and 
simple, that the mass of $\mu$ is concentrated at these zeros, and that the mass of $\mu$ at the zero $z$ is $C(\alpha, z) / S^{\prime}(\alpha, z)$. (7) and (8) now follow from the above properties of $\mu$. The equality (9) is easily verified from the equations (6) by a direct substitution. The inequality follows from (7) on choosing $\alpha$ so that $S(\alpha, t)=0$.

Proof of Lemma 3. Integrating both sides of (13) we have

$$
\frac{C(\alpha, z)}{S(\alpha, z)}-\frac{C(\alpha, w)}{S(\alpha, w)}=(w-z) \int \frac{d \mu(t)}{(t-z)(t-w)}+A(w-z) .
$$

Let $w$ be fixed, let $z=i y$, divide both sides with respect to $z$ and let $|y| \rightarrow \infty$. By the Lebesgue dominated convergence theorem,

$$
\frac{C(\alpha, i y)}{i y S(\alpha, i y)} \rightarrow-A
$$

and therefore

$$
\frac{e^{i \alpha} E(i y)}{i y S(\alpha, i y)} \rightarrow-A,
$$

and this implies (10). To prove (11), suppose that for some real number $\alpha, S(\alpha, i y)=o(E(i y))(y \rightarrow+\infty)$. From (6) we can easily verify that

$$
S(\beta, i y)=\sin (\alpha-\beta) e^{i \alpha} E(i y)+e^{i(\alpha-\beta)} S(\alpha, i y)
$$

and hence

$$
\lim _{y \rightarrow+\infty} S(\beta, i y) / E(i y)=\sin (\alpha-\beta) e^{i \alpha} .
$$

If $\beta$ is not congruent to $\alpha$ modulo $\pi, \sin (\alpha-\beta) \neq 0$ and

$$
S(\beta, i y) \neq o(E(i y)) \quad(y \rightarrow+\infty) .
$$

Proof of Lemma 4. If $\beta$ is congruent to $\alpha$ modulo $\pi$, (12) follows from (9). Suppose then that $\beta$ is not congruent to $\alpha$ modulo $\pi$. It is clear from the definitions that the zeros of $S(\alpha, z)$ are then distinct from the zeros of $S(\beta, z)$. Note that when $S(\alpha, t)=0, S(\beta, t)$ $=\sin (\beta-\alpha) C(\alpha, t)$ and that when $S(\beta, t)=0, S(\alpha, t)=\sin (\alpha-\beta) C(\beta, t)$. Therefore, (12) is equivalent to the formula

$$
\sum_{S(\alpha, t)=0} \frac{F_{1}(t)}{S^{\prime}(\alpha, t)(z-t)}=\frac{F_{1}(z)}{S(\alpha, z)}
$$

when $S(\beta, z)=0$. By $(8)$ and the hypotheses on $F_{1}(z)$ and the Schwarz inequality, the left hand side of (14) is absolutely convergent when- 
ever $S(\alpha, z) \neq 0$. We prove the lemma by showing that (14) is valid for all such complex $z$. Note that (1) implies that

$$
\int \frac{\log ^{+}|S(\alpha, t)| d t}{1+t^{2}}<\infty .
$$

It follows from (4) and (10) that

$$
\left|F_{1}(i y)\right|=o(y S(\alpha, i y)) \quad(|y| \rightarrow \infty) .
$$

By the proof of Lemma 2 of [2], there is a complex constant $B=B(\alpha)$ independent of $z$ such that

$$
\sum_{S(\alpha, t)=0} \frac{F_{1}(t)}{S^{\prime}(\alpha, t)(z-t)}=\frac{F_{1}(z)}{S(\alpha, z)}+B,
$$

or equivalently

$$
\frac{S(\alpha, z)}{E(z)} \sum_{S(\alpha, t)=0} \frac{F_{1}(t)}{S^{\prime}(\alpha, t)(z-t)}=\frac{F_{1}(z)}{E(z)}+B \frac{S(\alpha, z)}{E(z)} .
$$

By the Lebesgue dominated convergence theorem, when $z=i y$ and $y \rightarrow+\infty$, the sum above approaches zero. On the real axis, $|S(\alpha, z) / E(z)| \leqq 1$. Since (1) holds,

$$
\lim _{\nu \rightarrow+\infty} y^{-1} \log |E(i y)|
$$

exists (Boas [1, p. 96]). It follows from (2) that

$$
\limsup _{y \rightarrow+\infty} y^{-1} \log |S(\alpha, i y) / E(i y)| \leqq 0 .
$$

By Boas [1, p. 93], $|S(\alpha, z) / E(z)| \leqq 1$ for $y \geqq 0$. By hypothesis,

$$
\lim _{y \rightarrow+\infty} F_{1}(i y) / E(i y)=0 .
$$

Therefore, $\lim _{y \rightarrow+\infty} B S(\alpha, i y) / E(i y)=0$. Since by hypothesis (11) does not hold, $B=0$. Q.E.D.

Proof OF TheOREM. Let $F(z)$ satisfy the hypothesis of the theorem and suppose that the sum on the right hand side of (5) converges for some real $\alpha$ which does not satisfy (11). Let $\beta$ be real. Let $\lambda_{1}$ be any zero of $S(\beta, z)$ different from $\lambda$. By Lemma 4 with $F_{1}(z)=S(\beta, z)$ $\cdot\left(z-\lambda_{1}\right)^{-1}$

$$
2 i \sum_{S(\alpha, t)=0} \frac{1}{E(t) \bar{E}^{\prime}(t)-\bar{E}(t) E^{\prime}(t)} \frac{S(\beta, t)}{\left(t-\lambda_{1}\right)} \frac{S(\beta, t)}{(t-\lambda)}=0 .
$$

On the other hand, if $F_{1}(z)=S(\beta, z)(z-\lambda)^{-1}$, then Lemma 4 yields 


$$
\begin{gathered}
2 i \sum_{S(\alpha, t)=0} \frac{1}{E(t) \bar{E}^{\prime}(t)-\bar{E}(t) E^{\prime}(t)} \frac{S^{2}(\beta, t)}{(t-\lambda)^{2}} \\
=\frac{2 i\left(S^{\prime}(\beta, \lambda)\right)^{2}}{E(\lambda) \bar{E}^{\prime}(\lambda)-\bar{E}(\lambda) E^{\prime}(\lambda)} .
\end{gathered}
$$

By Lemma 4 with $F_{1}(z)=F(z)$ and Bessel's inequality (Halmos [3, p. 14])

$$
\begin{aligned}
& 2 i \sum_{S(\beta, t)=0} \frac{|F(t)|^{2}}{E(t) \bar{E}^{\prime}(t)-\bar{E}(t) E^{\prime}(t)} \\
& \quad \leqq 2 i \sum_{S(\alpha, t)=0} \frac{|F(t)|^{2}}{E(t) \bar{E}^{\prime}(t)-\bar{E}(t) E^{\prime}(t)} \\
& \quad<\infty .
\end{aligned}
$$

For real $x$, let $\phi(x)$ be the phase of $\bar{E}(x)$. Then

$$
\phi^{\prime}(x)=\frac{1}{2} i\left(\bar{E}(x) E^{\prime}(x)-E(x) \bar{E}^{\prime}(x)\right) /|E(x)|^{2}>0 .
$$

Therefore, $\phi(x)$ can be defined for all real $x$ so as to be a continuous increasing function of $x$. (15) can be written

$$
\sum_{S(\beta, t)=0}\left|\frac{F(t)}{E(t)}\right|^{2} \frac{1}{\phi^{\prime}(t)} \leqq 2 i \sum_{S(\alpha, t)=0} \frac{|F(t)|^{2}}{E(t) \bar{E}^{\prime}(t)-\bar{E}(t) E^{\prime}(t)} .
$$

Integrate each side from $\beta=0$ to $\beta=\pi$. Since the summand is nonnegative, the sum and the integral can be interchanged. Therefore,

$$
\int\left|\frac{F(t)}{E(t)}\right|^{2} d t \leqq 2 \pi i \sum_{S(\alpha, t)=0} \frac{|F(t)|^{2}}{E(t) \bar{E}^{\prime}(t)-\bar{E}(t) E^{\prime}(t)} .
$$

By Lemma 3, for all but one number $\beta$ modulo $\pi$, (11) does not hold. For such $\beta, \alpha$ and $\beta$ can be interchanged in (15) and hence the equality holds. It is clear that the equality must hold in (16) and hence (5) follows in this case.

Suppose on the other hand that $F(z)$ satisfies the hypotheses of the theorem and that the left hand side of (5) converges. As we have just seen,

$$
\int\left|\frac{F(t)}{E(t)}\right|^{2} d t=2 \pi i \int_{\alpha=0}^{\alpha=\pi} d \alpha \sum_{S(\alpha, t)=0} \frac{|F(t)|^{2}}{E(t) \bar{E}^{\prime}(t)-\bar{E}(t) E^{\prime}(t)}<\infty .
$$


Therefore, for more than one real $\alpha$ modulo $\pi$, the right hand side of (5) converges. If we choose $\alpha$ so as not to satisfy (11), then (5) follows by the first part of the proof. Q.E.D.

\section{REFERENCES}

1. R. P. Boas, Jr., Entire functions, New York, Academic Press, 1954.

2. L. de Branges, The Bernstein problem, Proc. Amer. Math. Soc. vol. 10 (1959) pp. 825-832.

3. P. R. Halmos, Introduction to Hilbert space and spectral multiplicity, New York, Chelsea, 1951.

4. L. H. Loomis and D. V. Widder, The Poisson integral representation of functions which are positive and harmonic in a half-plane, Duke Math. J. vol. 9 (1942) pp. 643645.

Lafayette College 